\title{
Utilização de metodologias ativas no curso de graduação em Enfermagem: uma oportunidade de superação do modelo de ensino tradicional
}

Use of active methodologies in the Nursing graduate course: an opportunity to overcome the traditional teaching model

Uso de metodologías activas en lo curso de grado en Enfermería: una oportunidad para superar el modelo de enseñanza tradicional

Francisco Lucas de Lima Fontes ORCID: https://orcid.org/0000-0003-1880-9329

Faculdade Evangélica do Meio Norte, Brasil

E-mail: lucasfontesenf@ufpi.edu.br

Alexsandra Maria Ferreira de Araújo Bezerra

ORCID: https://orcid.org/0000-0002-9306-581X Sociedade Brasileira de Terapia Intensiva, Brasil

E-mail: alexsandrabezerra@ rocketmail.com

Hallyson Leno Lucas da Silva

ORCID: https://orcid.org/0000-0001-7237-1706

Faculdades Integradas de Patos, Brasil

E-mail: hallyson.silva@ hapvida.com.br

Ilana Maria Brasil do Espírito Santo

ORCID: https://orcid.org/0000-0001-9591-2704

Centro Universitário Internacional, Brasil E-mail: ilaleao@outlook.com

Thalyta Maria Campelo Marques ORCID: https://orcid.org/0000-0001-9978-8659 Faculdade UNINASSAU, Brasil

E-mail: talitamarkes1@hotmail.com

Mauricio José Almeida Morais ORCID: https://orcid.org/0000-0002-6451-4284 Instituto de Ensino Superior Múltiplo, Brasil E-mail: maurjose64@gmail.com

Reberson do Nascimento Ribeiro ORCID: https://orcid.org/0000-0003-2644-9917

Centro Universitário UNIFACID, Brasil E-mail: rebersonsrn98@gmail.com Deylane de Melo Barros

ORCID: https://orcid.org/0000-0002-4803-4694 Centro Universitário UNINOVAFAPI, Brasil E-mail: anemelob@gmail.com

Mauro Sérgio Mendes Dantas ORCID: https://orcid.org/0000-0001-9521-7867

Salve Vidas - Cursos e Treinamentos, Brasil

E-mail: maurosergio180@hotmail.com

Márcia Sandra Rêgo de Sousa ORCID: https://orcid.org/0000-0003-3495-3865 Centro Universitário UNINOVAFAPI, Brasil E-mail: marciasandrego@gmail.com

Nayla Karine Barros da Silva ORCID: https://orcid.org/0000-0003-2959-1433 Instituto de Ensino Superior Múltiplo, Brasil E-mail: naylabarrosg1@gmail.com

Sandra Valéria Nunes Barbosa ORCID: https://orcid.org/0000-0003-4281-8807 Faculdade FAVENI, Brasil E-mail: sandranunesb79@gmail.com Mayara Macêdo Melo

ORCID: https://orcid.org/0000-0001-8144-7653 Universidade Federal do Piauí, Brasil E-mail: mayaramelo@ufpi.edu.br 


\begin{abstract}
Resumo
Objetivo: Compreender a utilização de metodologias ativas no curso de graduação em Enfermagem. Metodologia: Trata-se de uma revisão integrativa de literatura que teve como questão norteadora "Quais os benefícios que a utilização de metodologias ativas no curso de graduação em Enfermagem traz para a superação do modelo de ensino tradicional?". A revisão foi construída por meio da Biblioteca Virtual em Saúde e do Portal de Periódicos da Coordenação de Aperfeiçoamento de Pessoal de Nível Superior, utilizando-se os descritores "metodologias ativas", "ensino" e "Enfermagem". Resultados: No que diz respeito aos temas abordados pelos artigos selecionados para discussão, houve predominância de debates sobre o processo de ensino-aprendizagem, práticas de monitoria acadêmica, dificuldades de ensino, planejamento docente e desempenho de estudantes. Quanto às metodologias ativas comumente utilizadas nos estudos, destacaram-se a aprendizagem baseada em problemas, a metodologia problematizadora e a análise das metodologias ativas no geral. Considerações finais: Infere-se que a utilização de metodologias ativas no curso de graduação em Enfermagem leva a formação de um enfermeiro crítico-reflexivo. Por meio delas, torna-se possível que o aluno se conscientize de suas qualidades e limitações na busca por melhores estratégias de aprendizagem. O emprego dessas metodologias rompe com o modelo tradicional de ensino, por tanto tempo empregado nas instituições formadoras.
\end{abstract}

Palavras-chave: Enfermagem; Ensino; Metodologias ativas.

\begin{abstract}
Objective: To understand the use of active methodologies in the Nursing graduate course. Methodology: This is an integrative literature review that had as its guiding question "What are the benefits that the use of active methodologies in the Nursing graduate course brings to overcome the traditional teaching model?". The review was constructed through the Biblioteca Virtual em Saúde and the Portal de Periódicos da Coordenação de Aperfeiçoamento de Pessoal de Nível Superior, using the descriptors "metodologias ativas", "ensino" and "Enfermagem". Results: With regard to the topics covered by the articles selected for discussion, there was a predominance of debates about the teaching-learning process, academic tutoring practices, teaching difficulties, teacher planning and student performance. As for the active methodologies commonly used in the studies, the problem-based learning, the problematizing methodology and the analysis of the active methodologies in general stood out. Final considerations: It is inferred that the use of active methodologies in the Nursing course leads to the formation of a critical-reflective nurse. Through them, it becomes possible for the student to become aware of his qualities and limitations in the search for better learning strategies. The use of these methodologies breaks with the traditional model of teaching, used for so long in educational institutions.
\end{abstract}

Keywords: Nursing; Teaching; Active methodologies.

\title{
Resumen
}

Objetivo: Comprender el uso de metodologías activas en lo curso de grado en Enfermería. Metodología: Se trata de una revisión integradora de la literatura que tuvo como pregunta orientadora "¿Cuáles son los beneficios que aporta el uso de metodologías activas en lo curso de grado en Enfermería para superar el modelo de enseñanza tradicional?". La revisión se construyó a través de la Biblioteca Virtual em Saúde y el Portal de Periódicos da Coordenação de Aperfeiçoamento de Pessoal de Nível Superior, utilizando los descriptores "metodologias ativas", "enseñanza" y "Enfermería". Resultados: En cuanto a los temas tratados por los artículos seleccionados para discusión, predominaron los debates sobre el proceso de enseñanza-aprendizaje, las prácticas de tutoría académica, las dificultades docentes, la planeamento docente y el desempeño de los estudiantes. En cuanto a las metodologías activas comúnmente utilizadas en los estudios, se destacó el aprendizaje basado en problemas, la metodología problematizadora y el análisis de las metodologías activas en general. Consideraciones finales: Se infiere que el uso de metodologías activas en lo curso de grado en Enfermería conduce a la formación de una enfermera crítico-reflexiva. A través de ellos, se hace posible que el alumno tome conciencia de sus cualidades y limitaciones en la búsqueda de mejores estrategias de aprendizaje. El uso de estas metodologías rompe con el modelo tradicional de enseñanza, utilizado durante tanto tiempo en las instituciones educativas.

Palabras clave: Enfermería; Enseñanza; Metodologías activas.

\section{Introdução}

O processo formativo dos profissionais da saúde tem sido pauta de discussão e críticas pelo uso de metodologias tradicionais que fragmentam o ensino. A procura pela eficiência técnica e pelo conhecimento específico colaboraram por anos para a solidificação da educação bancária, aquela em que o professor se assume na postura de "transmissor do conhecimento" e seu aluno admite-se na condição de agente passivo, receptor desse saber (Costa et al., 2015; Fontes et al., 2020).

Apesar disso, mudanças sociais contemporâneas têm contribuído para a discussão aprofundada sobre a dinâmica de ensino-aprendizagem necessária à formação de novos profissionais da saúde e para a construção de conhecimento relevante ao 
contexto das profissões, de modo a conceber um saber-fazer científico temporário (Mitre et al., 2008).

Para que tais transformações ocorram, instituições de ensino superior precisam revisar o processo de ensinoaprendizagem e incentivar o protagonismo do aluno na interdisciplinaridade com a estruturação de redes sociais distintas do modelo de ensino por tempos praticado, onde prevalecia hábitos alienantes e tecnicistas (Xavier et al., 2014; Fontes et al., 2019).

A excelência na oferta de cuidados é tida como central na formação do enfermeiro, de modo que, para o alcance de tal primazia é necessária a promoção não apenas de habilidades técnicas e científicas, mas também de competências relacionais. A Enfermagem é uma profissão que ainda constrói sua estrutura, justamente por isso há uma inquietude excessiva com a especialidade, valorizando o conhecimento técnico-científico. Esse fato é evidenciado por ênfase em aspectos biologicistas no processo saúde-doença presentes no curso de graduação. Outros aspectos como socioeconômicos e psicoativos são esquecidos e não abordados, o que provoca distanciamento dos debates de caráter moral (Santos, 2005; Fontes et al., 2019; Santos et al., 2020).

Assim, a procura por métodos inovadores na dinâmica do ensino-aprendizagem surge para contemplar as reais necessidades da sociedade moderna, de modo a superar limites do preparo meramente técnico para, definitivamente, haver aproximação com a formação dialógica baseada na ação-reflexão-ação (Mitre et al., 2008).

$\mathrm{Na}$ busca por essas mudanças, evidencia-se a utilização de métodos de aprendizagem ditos "ativos", que se amparam no discurso do ensino problematizador. Dessa forma, entra em ação a escolha de metodologias ativas como recursos de ação educativa que instigam procedimentos de ensino-aprendizagem crítico-reflexivos, oportunizando ao discente o protagonismo na construção de seu conhecimento (Rodrigues \& Caldeira, 2008).

Em seu estudo, Chaves et al. (2020) discutem as metodologias ativas comumente empregadas mediante o conteúdo teórico programado, evidenciando algumas das metodologias viáveis a aplicação no ensino da Enfermagem como a aprendizagem baseada em problemas (problem based learning), aprendizagem entre pares ou grupos (team based learning), estudos de caso, sala de aula invertida (flipped classroom), treinamento de habilidades técnicas, simulações, metodologia problematizadora, aprendizagem baseada em jogos e projetos, dramatização, dentre outras.

Para que o uso dessas metodologias ativas seja efetivo é necessário que o enfermeiro, enquanto docente, se utilize de estratégias que o norteie na melhoria e no desenvolvimento de suas práticas pedagógicas. Atividades como atualização em conteúdos e práticas, participação em cursos e eventos científicos para aprimoramento, leitura constante, escuta ativa ao alunato, pesquisa do feedback discente acerca de disciplinas e conteúdos ministrados, produção científica e estímulo ao raciocínio crítico do aluno são ações que fortalecem a utilização e implementação de metodologias ativas em sala de aula (Fontes et al., 2019; Dias et al., 2020).

No estudo das metodologias ativas no ensino da Enfermagem um ponto deve ser levado em consideração: os enfermeiros que atuam como docentes comumente foram graduados em um modelo de ensino tradicional, pautado no professor como único protagonista e detentor de todo o saber. Para que ocorra a ruptura desse cenário faz-se necessário o correto direcionamento quanto ao preparo e uso desses relevantes recursos didático-pedagógicos. A utilização das metodologias ativas na graduação em Enfermagem tornou-se uma realidade no mundo contemporâneo e seus benefícios são relevantes ao ensino crítico-reflexivo, com foco no encorajamento do aluno na construção de seu saber, aprendizado escrito e oral expressivo, resolução de problemas e ganho de autonomia no enfrentamento de situações que envolvem a tomada de decisões na profissão. Diante do que foi exposto, o objetivo do presente estudo foi compreender a utilização de metodologias ativas no curso de graduação em Enfermagem. 


\section{Metodologia}

Trata-se de uma revisão integrativa de literatura. A questão norteadora que este estudo levantou foi a seguinte: "Quais os benefícios que a utilização de metodologias ativas no curso de graduação em Enfermagem traz para a superação do modelo de ensino tradicional?".

A revisão foi construída por meio da Biblioteca Virtual em Saúde (BVS) e do Portal de Periódicos da Coordenação de Aperfeiçoamento de Pessoal de Nível Superior (Portal da CAPES). Foram incluídos na pesquisa apenas artigos originais e relatos de experiência que tinham como objeto central de estudo as metodologias ativas e sua relação com a graduação em Enfermagem, com delimitação temporal de 2014 a 2019, disponíveis na íntegra, de maneira gratuita e em língua portuguesa. Foram excluídas monografias, dissertações e teses.

Utilizaram-se os seguintes Descritores em Ciências da Saúde (DeCS) para busca dos estudos: "metodologias ativas", "ensino" e "Enfermagem". Com a finalidade de sistematizar e otimizar a busca, optou-se por utilizar o operador booleano "AND", cruzando o descritor "metodologias ativas" com os demais. A partir da utilização dos descritores acima citados, foi encontrado um total de 190 estudos (Figura 1). A seleção dos estudos que cumpriam com os critérios de inclusão e comporiam essa revisão foi realizada por meio de leitura e análise crítica dos resumos. Ao final do processo, 10 artigos correspondiam ao objetivo proposto.

Figura 1. Etapas de seleção dos estudos.

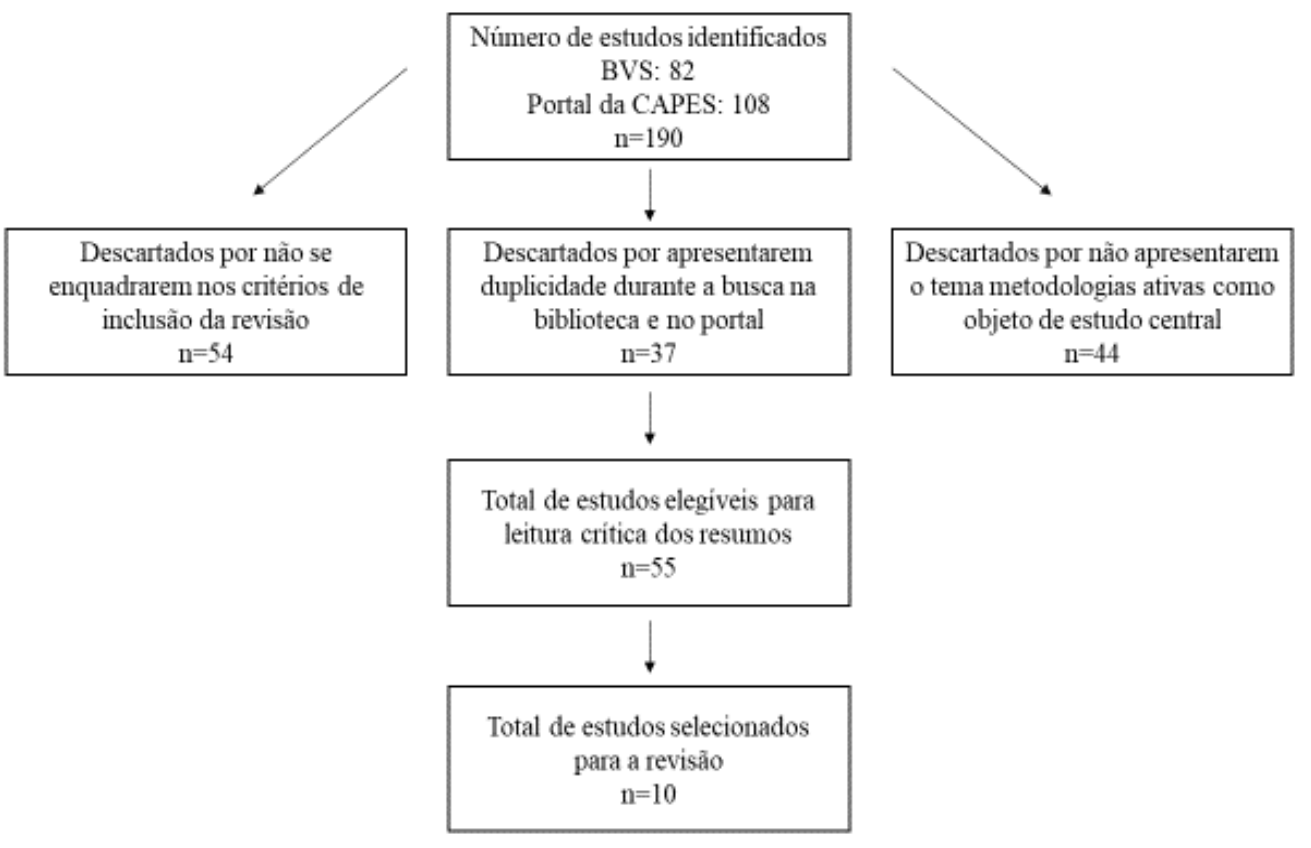

Fonte: Autores (2021).

\section{Resultados}

No intento de facilitar a visualização das principais características dos estudos selecionados, buscou-se organizar os aspectos das 10 pesquisas ancoradas para discussão. Na Tabela 1, pode ser visualizado o tipo de artigo, a abordagem metodológica, a tipo de estudo, as fontes e os periódicos nos quais os artigos foram publicados.

Referente ao tipo de artigo, 50\% eram artigos originais. A abordagem metodológica mais frequente foi a qualitativa (80\%). No recorte, os estudos descritivos foram vistos em $70 \%$ das publicações. Concernente às fontes, $70 \%$ dos artigos foram 
encontrados na BVS. Já em relação aos periódicos, 30\% dos artigos selecionados foram publicados pela Revista de Enfermagem da UFPE.

No que diz respeito aos temas abordados pelos artigos selecionados, predominou o debate sobre o processo de ensinoaprendizagem (40\%), tendo também as práticas de monitoria acadêmica uma parcela de discussão (20\%). Quanto as metodologias ativas comumente utilizadas nos estudos, $10 \%$ se ancoraram na aprendizagem baseada em problemas, $20 \%$ fizeram uma análise das metodologias ativas no geral, $30 \%$ adotaram a metodologia problematizadora e $40 \%$ empregaram outros métodos ativos. Esses dados estão expostos na Tabela 2. De modo a sintetizar os resultados principais dos estudos, elaborou-se a Tabela 3, explorando as principais ideias encontradas na conclusão dos 10 estudos selecionados para compor a discussão desta revisão.

Tabela 1. Caracterização dos estudos publicados e fontes de publicação (2014-2019).

\begin{tabular}{|c|c|}
\hline Tipos de artigos & Quantidade \\
\hline Artigo original & 05 \\
\hline Relato de experiência & 05 \\
\hline TOTAL & 10 \\
\hline \multicolumn{2}{|l|}{ Abordagem metodológica } \\
\hline Qualitativo & 08 \\
\hline Quantitativo & 01 \\
\hline Misto & 01 \\
\hline TOTAL & 10 \\
\hline \multicolumn{2}{|l|}{ Tipos de estudos } \\
\hline Descritivo & 07 \\
\hline Exploratório & 00 \\
\hline Descritivo e exploratório & 03 \\
\hline TOTAL & 10 \\
\hline \multicolumn{2}{|l|}{ Fontes dos estudos } \\
\hline BVS & 07 \\
\hline Portal da CAPES & 03 \\
\hline TOTAL & 10 \\
\hline \multicolumn{2}{|l|}{ Periódicos } \\
\hline Revista Acta Paulista de Enfermagem & 01 \\
\hline Revista Baiana de Enfermagem & 01 \\
\hline $\begin{array}{l}\text { Revista da Faculdade de Ciências Médicas de } \\
\text { Sorocaba }\end{array}$ & 01 \\
\hline Revista de Enfermagem da UFPE & 03 \\
\hline Revista Escola Anna Nery & 01 \\
\hline Revista Mineira de Enfermagem & 01 \\
\hline Revista Pró-univerSUS & 01 \\
\hline Revista Trabalho, Educação e Saúde & 01 \\
\hline TOTAL & 10 \\
\hline
\end{tabular}

Fonte: Autores (2021). 
Tabela 2. Organização dos estudos quanto aos temas abordados e metodologias ativas utilizadas (2014-2019).

\begin{tabular}{lc}
\hline \multicolumn{1}{c}{ Temas abordados } & Quantidade \\
\hline Desempenho de estudantes & 01 \\
Dificuldades de ensino & 01 \\
Monitoria acadêmica & 02 \\
Planejamento docente & 01 \\
Prática docente & 01 \\
Processo de ensino-aprendizagem & 04 \\
TOTAL & 10 \\
\hline \multicolumn{2}{c}{ Metodologias ativas utilizadas } \\
\hline Análise de metodologias ativas & 02 \\
Aprendizagem baseada em problemas & 01 \\
Metodologia problematizadora & 03 \\
Outras & 04 \\
TOTAL & 10 \\
\hline
\end{tabular}

Fonte: Autores (2021).

Tabela 3. Conclusão dos estudos sobre metodologias ativas selecionados para a discussão da revisão (2014-2019).

\begin{tabular}{|c|c|}
\hline Melo et al. (2014) & $\begin{array}{l}\text { É importante destacar que para um curso ancorado na Aprendizagem Baseada em } \\
\text { Problemas seja eficaz é preciso constante discussão e reflexão entre docentes e discentes } \\
\text { sobre novas estratégias que possam minimizar as dificuldades enfrentadas pelos alunos } \\
\text { que trabalham para uma aprendizagem efetiva }\end{array}$ \\
\hline $\begin{array}{l}\text { Mesquita, Meneses \& } \\
\quad \text { Ramos (2016) }\end{array}$ & $\begin{array}{l}\text { Compreender a utilização de metodologias ativas é fundamental para atender aos } \\
\text { pressupostos do paradigma educacional contemporâneo }\end{array}$ \\
\hline Carvalho et al. (2016) & $\begin{array}{l}\text { A atividade desenvolvida fortaleceu os conhecimentos acerca dos métodos } \\
\text { problematizadores de ensino, despertou a reflexão sobre o planejamento na prática } \\
\text { docente e contribuiu para desconstruir um "olhar" limitado sobre o processo de ensino- } \\
\text { aprendizagem, antes norteado pela formação tradicional das participantes }\end{array}$ \\
\hline Brito et al. (2017) & $\begin{array}{l}\text { A experiência na atividade de estímulo ao ensino docente proporcionou a fusão de } \\
\text { conhecimentos pela elaboração e realização de metodologias ativas e pela convivência } \\
\text { com professores e alunos que contribuíram para a formação profissional e consolidaram } \\
\text { o desenvolvimento de ações essenciais à vida universitária }\end{array}$ \\
\hline Mendonça et al. (2017) & $\begin{array}{l}\text { Faz-se necessário transformar o aluno em protagonista de sua aprendizagem, para que } \\
\text { seja desenvolvido o senso crítico diante do que é aprendido e competências para } \\
\text { relacionar os conhecimentos construídos ao que será vivenciado no mundo real }\end{array}$ \\
\hline Fabbro et al. (2018) & $\begin{array}{l}\text { Os caminhos trilhados pela disciplina mostram a necessidade constante de avaliação da } \\
\text { prática docente, dado que somente na reflexão e autorreflexão que se possibilita o } \\
\text { movimento necessário e motivante, de não somente "fazer diferente", mas também } \\
\text { "fazer melhor", do ponto de vista da avaliação constante da prática docente, bem como } \\
\text { da formação do aluno como ser humano }\end{array}$ \\
\hline Macedo et al. (2018) & $\begin{array}{l}\text { Há perspectivas de inclusão de metodologias ativas de aprendizagem na prática dos } \\
\text { docentes, podendo tornar-se estratégia pedagógica e atender às Diretrizes Curriculares } \\
\text { Nacionais nesse cenário }\end{array}$ \\
\hline $\begin{array}{l}\text { Pascon, Otrenti \& Mira } \\
\text { (2018) }\end{array}$ & $\begin{array}{l}\text { A prova de progressão avalia o desempenho do estudante longitudinalmente, identifica } \\
\text { potencialidades e fragilidades curriculares, além de avaliar a instituição. Compreende } \\
\text { um dos instrumentos avaliativos no uso de metodologias ativas de ensino e deve ser } \\
\text { incentivada quanto à sua aplicabilidade nos cursos de graduação }\end{array}$ \\
\hline Felix \& Soares (2019) & $\begin{array}{l}\text { Observa-se que o uso de abordagens inovadoras no ensino de Enfermagem em doenças } \\
\text { transmissíveis tornou o aprendizado mais dinâmico e atraente. Faz-se esse conhecimento } \\
\text { essencial para que os aprendizes desenvolvam competências básicas para atender } \\
\text { clientes com melhor qualidade assistencial e segurança }\end{array}$ \\
\hline $\begin{array}{l}\text { Freitas \& Santos } \\
\qquad(2019)\end{array}$ & $\begin{array}{l}\text { Contribui-se, pelo uso das metodologias ativas, das oficinas e dos modelos didáticos } \\
\text { construídos na monitoria, na formação dos discentes, sendo eficaz seu uso no ambiente } \\
\text { acadêmico; a monitoria contribui na formação de competências e habilidades dos futuros } \\
\text { profissionais de Enfermagem, que tanto precisam da aproximação entre teoria e prática } \\
\text { para o aperfeiçoamento de suas tomadas de decisões, muitas vezes, em situações críticas }\end{array}$ \\
\hline
\end{tabular}




\section{Discussão}

O enfermeiro docente que atua na formação de outros profissionais comumente foi formado por uma metodologia de ensino tradicional. Boa parte desses profissionais não possui licenciatura ou qualquer curso de formação docente que o aproxime de métodos pedagógicos necessários ao ensino. De modo a assistir e se adequar às transformações do processo formativo e tornar a dinâmica de aprendizagem significativa é importante um olhar diferenciado com vistas à inserção de uma nova maneira de ensinar pelo docente, aproximando o aluno da realidade que será vivida profissionalmente (Mendonça et al., 2017).

A procura por enfermeiros docentes qualificados e alinhados às orientações pedagógicas transformadoras tornou-se necessária com a identificação de métodos ultrapassados nas práticas de ensino. Com isso, percebeu-se nos últimos anos a adoção de estratégias de aprendizagem problematizadoras sendo incorporadas aos planos de ensino e cronogramas das disciplinas dos cursos de graduação em Enfermagem. A mudança nas grades curriculares dos cursos solidificou a ideia de que o ensino necessita ser reinventado por meio da introdução de tecnologias e inovações que auxiliem o aluno a tornar-se mais ativo na dinâmica de aprendizagem. Esse processo intensifica o caminho para modernização das metodologias, seja por meio da problematização, gamificação, estudos de caso, treinamento de habilidades, autoavaliação ou grupos de discussão (Carvalho et al., 2016).

$\mathrm{O}$ enfermeiro professor está inserido em um quadro que requer alternativas para a prática transformadora e multiplicadora. Entende-se que a educação funciona por meio de importante fonte de recursos pedagógicos que favorece a formação ampla do profissional, fato evidenciado pela necessidade constante de transformação das estratégias didáticas úteis à melhoria do ensino-aprendizagem. Essas estratégias delineiam o profissional que será colocado no mercado de trabalho e a qualidade do atendimento prestado no cumprimento das competências inerentes à profissão (Mendonça et al., 2017).

A preparação do discente para a aprendizagem dos conteúdos relevantes a sua construção profissional precisa da produção de situação que incentive sua capacidade de pensar, uma vez que é baseado na interação do aluno com o meio que a aprendizagem se constrói e o "pensar" se desenvolve (Brito et al., 2017). Melo et al. (2014) pontuam ainda que, para que esse preparo do discente ocorra efetivamente, é necessário que o docente tenha tido uma boa formação, de maneira que a relação aluno-professor seja colaborativa não apenas em termos de conhecimentos que podem ser oferecidos, mas também por meio das vivências e trajetórias do enfermeiro docente.

Dentro da gama de instrumentos facilitadores desse vínculo aluno-professor estão as metodologias ativas. Para a Enfermagem, a aquisição de competências docentes no emprego desses métodos atravessa o conflito entre ensino passivo versus ensino ativo, com mobilização das instituições de ensino frente a novas metodologias e tecnologias educacionais. A aplicação dessas metodologias promove a educação crítica-reflexiva baseada em estímulo no processo de ensinoaprendizagem, o que resulta em envolvimento por parte do discente na busca pelo conhecimento. Esses recursos proporcionam ao aluno refletir conteúdos, buscar resultados para problemas criados nas atividades e propor soluções pertinentes às situações (Macedo et al., 2018).

A introdução de metodologias ativas no ensino, a começar pelo planejamento docente, pode ser tida como uma substituição ao ensino tradicional para uma educação baseada em competências e habilidades. A aplicação das metodologias ativas deve ocorrer ainda na reestruturação da disciplina, com introdução de novas concepções de saúde e de educação no norteio de caminhos viáveis à formação profissional (Carvalho et al., 2016).

Um dos aspectos positivos proporcionados pelo uso das metodologias ativas relaciona-se à articulação entre teoria $\mathrm{e}$ prática, além da necessidade de retomada de outras temáticas, pois ao passo em que se vivencia a prática no campo, tem-se a oportunidade de constatar lacunas. Os debates realizados no decorrer da prática clínica possibilitam uma concepção ampliada da função do enfermeiro, indo além da abordagem biologicista, o que releva a importância tanto de tanto de dimensões técnicas 
quanto de perspectivas associadas à educação em saúde, valorizando a função educativa do enfermeiro (Fabbro et al., 2018).

Concernente às metodologias ativas no ensino-aprendizagem, merece destaque o uso da aprendizagem baseada em problemas fundamentada em situação-problema. No uso dessa metodologia, o aprendiz compreende termos e expressões do problema, o define, analisa-o, organiza hipóteses de explicação ou solução, elabora propostas de aprendizagem, define bases de informações para aquisição de novos conhecimentos, sintetiza tais conhecimentos e revisa as hipóteses formuladas para o problema. Esse recurso coloca o discente como protagonista de seu aprendizado e estimula a proatividade (Fabbro et al., 2018).

A metodologia da aprendizagem baseada em problemas possui forte relação com a aprendizagem significativa, que sustenta que todo novo conhecimento pode ser significativo quando interrelacionado com saberes prévios dos discentes. Considera-se relevante o senso comum interpelado pelo conhecimento científico, em um diálogo contínuo na transformação do ensino e da aprendizagem (Pascon, Otrenti \& Mira, 2018). Por sua vez, a metodologia problematizadora ancora-se no referencial proposto por Paulo Freire, cuja concepção baseia-se em um ensino libertador, dialógico, reflexivo, conscientizador, transformador e crítico, onde os problemas surgem a partir de uma realidade. Essa metodologia é, dentre as estratégias ativas de ensino, a mais utilizada (Macedo et al., 2018).

Além da metodologia problematizadora e da aprendizagem baseada em problemas, outras estratégias ativas podem ser empregadas no ensino da Enfermagem, dentre elas a sala de aula invertida (flipped classroom), a simulação, o mapa conceitual, a storytelling (narração de história) e a aprendizagem baseada em jogos, descritas no Quadro 1.

Quadro 1. Metodologias ativas empregadas no ensino da Enfermagem.

\begin{tabular}{|c|c|}
\hline METODOLOGIAS & DINÂMICA DE OCORRÊNCIA \\
\hline Sala de aula invertida (flipped classroom) & $\begin{array}{l}\text { A abordagem de aprendizagem em grupo se altera para a } \\
\text { aprendizagem individual. O ensino se torna dinâmico e interativo a } \\
\text { medida em que o educador norteia os alunos a aplicarem conceitos e } \\
\text { se envolverem criativamente nos assuntos. Comumente, eventos que } \\
\text { aconteciam internamente em sala passam a ocorrer fora da sala de } \\
\text { aula }\end{array}$ \\
\hline Simulação & $\begin{array}{l}\text { Método utilizado para substituir ou aumentar experiências reais em } \\
\text { experiências dirigidas, reproduzindo perspectivas importantes do } \\
\text { mundo real de maneira interativa. Podem ser utilizados manequins } \\
\text { estáticos, robôs e pacientes padronizados (atores) }\end{array}$ \\
\hline Mapa conceitual & $\begin{array}{l}\text { Esquema que utiliza diversas correntes gráficas para interligar } \\
\text { conceitos. UUtil na compreensão de conteúdos por meio da } \\
\text { organização de informações e suas relações }\end{array}$ \\
\hline Narração de história (storytelling) & $\begin{array}{l}\text { Baseia-se na descrição de um fato ou evento que produz uma } \\
\text { lembrança na consciência do discente. No ensino da Enfermagem, a } \\
\text { utilização da storytelling encoraja o pensamento crítico-reflexivo, } \\
\text { bem como amplia a retenção de saberes e resolução de problemas. } \\
\text { Ademais, facilita a associação entre teoria e prática }\end{array}$ \\
\hline Aprendizagem baseada em jogos & $\begin{array}{l}\text { Fundamentam-se em um instrumento de ensino-aprendizagem eficaz, } \\
\text { em virtude do incentivo ao envolvimento e aumento da motivação do } \\
\text { discente, transformando a aprendizagem mais agradável. A aplicação } \\
\text { de jogos em sala de aula preenche lacunas entre teoria e prática, } \\
\text { provocando uma aprendizagem significativa }\end{array}$ \\
\hline
\end{tabular}

Fonte: Felix \& Soares (2019).

Semelhante à simulação prática, a construção de modelos didáticos foi vista no estudo de Freitas e Santos (2019). Essa metodologia de ensino valoriza as ideias e a criatividade do aluno. Os materiais foram construídos durante a monitoria e simulavam a Anatomia do corpo, com mãos feitas de luvas de látex para treinos de punção venosa periférica e músculos de regiões como a do deltoide e dorso-glútea, criados por meio de massa de EVA, para simularem a administração de medicamentos. Essa metodologia oportuniza ao discente uma realidade de como se dá a realização da técnica, uma vez que os 
materiais mostraram-se bastante realistas.

Outras metodologias ativas também podem ser empregadas, como a que Pascon, Otrenti \& Mira (2018) utilizaram em seu estudo. A prova de progressão, também conhecida como prova progressiva, ampara-se em uma avaliação composta por questões de múltipla escolha elaborada por meio dos conteúdos das diversas áreas que integram o currículo do curso. Esse tipo de prova possibilita ao aluno o conhecimento real de desempenho e progresso ao longo dos anos de curso. Apresenta-se como importante recurso diagnóstico do aprendizado sobre as áreas do currículo formativo do discente.

Mesmo com as evidentes vantagens de utilização das metodologias ativas no ensino da Enfermagem, enfermeiros docentes ainda encontram obstáculos para sua aplicação. Tais desafios relacionam-se a problemas curriculares que dificultam o emprego das metodologias inovadoras no ensino-aprendizagem, a resistência dos professores na implementação das metodologias e a não compreensão de aplicabilidade das estratégias ativas na prática docente (Mesquita, Meneses \& Ramos, 2016).

São necessários, portanto, que dois pontos sejam levantados, o primeiro associa-se aos cursos de graduação em Enfermagem. Estes devem introduzir estratégias ativas de ensino-aprendizagem em suas grades curriculares e estimular e capacitar os professores quanto ao seu uso, tendo em vista os diversos benefícios que elas trazem ao processo formativo do profissional (Felix \& Soares, 2019).

O segundo ponto diz respeito a importância de o docente estar sensível às necessidades do aluno, respeitando sua autonomia e criatividade e compreendendo que é mais válido para o discente descobrir um método de aprendizado do que receber do educador informações "mastigadas" acerca de conhecimentos científicos disponíveis. Deve ser levado em consideração que o indivíduo é ativo em sua essência e sua inteligência é construída por meio das relações com os pares e com o meio em que está inserido (Brito et al., 2017).

Apesar de as metodologias ativas serem um recurso reconhecidamente benéfico, faz-se necessário que docentes e discentes acreditem em seu potencial pedagógico e se esforcem a trabalhar intelectualmente e atenciosamente na construção do aprendizado. O encorajamento e empatia do professor facilita a aprendizagem do aluno. É importante salientar, contudo, que as metodologias sozinhas não são capazes de desenvolver a motivação autônoma do discente, sendo necessário estímulo e compreensão no uso de estratégias ativas para a dinâmica de ensino-aprendizagem (Macedo et al., 2018).

\section{Considerações Finais}

Infere-se que a utilização de metodologias ativas na graduação em Enfermagem leva a formação de um enfermeiro crítico-reflexivo. Por meio delas, torna-se possível que o aluno se conscientize de suas qualidades e limitações na busca por melhores estratégias de aprendizagem. O emprego dessas metodologias rompe com o modelo tradicional de ensino, por tanto tempo empregado nas instituições formadoras.

Contudo, essa ruptura tem de ser feita sem a criação de dificuldades. Apesar dos diversos benefícios que as metodologias ativas trazem ao processo de aprendizagem do discente, as instituições de ensino superior estão pouco preparadas para implementação desses recursos na prática pedagógica e docente. É necessária adequada reformulação das grades curriculares do curso de Enfermagem e das ementas de disciplinas, de modo a possibilitar que as metodologias sejam inseridas corretamente, distanciando-se paulatinamente no modelo tradicional de ensino.

Existem variadas metodologias e estas podem ser utilizadas de acordo com as situações, disciplinas, conteúdos e características dos alunos envolvidos. Além disso, espera-se que enfermeiros docentes compreendam a importância de implementação das metodologias ativas em sala de aula para formação de seu alunato, tendo a empatia, a criatividade, o encorajamento e o incentivo à autonomia como principais recursos na vivência deste campo de atuação profissional. 


\section{Referências}

Brito, L. S., Ribeiro, L. S., Ulisses, L. O., Ortiz, M. F. A. \& Whitaker, M. C. O. (2017). Experiência de discentes de enfermagem em metodologias ativas na atividade de ensino docente. Revista Baiana de Enfermagem, 31(3), e21715. http://doi.org/10.18471/rbe.v31i3.21715

Carvalho, A. C. O., Soares, J. R., Maia, E. R., Machado, M. F. A. S., Lopes, M. S. V. \& Sampaio, K. J. A. J. (2016). O planejar docente: relato sobre uso de métodos ativos no ensino de Enfermagem. Revista de Enfermagem da UFPE, 10(4), 1332-1338. https://doi.org/10.5205/1981-8963-v10i4a11121p1332-13382016

Chaves, U. S. B., Martins, A. S., Costa, C. C. P., Bisagni, C., Vieira, M. L. C. \& Jesus, P. B. R. (2020). Relato de experiência da utilização de metodologias ativas na prática da monitoria de um curso de Enfermagem. Research, Society and Development, 9(9), e316997303. http://doi.org/10.33448/rsd-v9i9.7303

Costa, R. R. O., Medeiros, S. M., Martins, J. C. A., Menezes, R. M. P. \& Araújo, M. S. (2015). O uso da simulação no contexto da educação e formação em saúde e enfermagem: uma reflexão acadêmica. Revista Espaço para a Saúde, 16(1), 59-65. http://doi.org/10.22421/1517-7130.2015v16n1p59

Dias, M. A. M., Oliveira, A. N. H., Souza, J. S., Rosa, F. T., Maia, T. S. C. \& Belarmino, L. M. (2020). Domínio das metodologias ativas por docentes de curso de graduação em Enfermagem. Research, Society and Development, 9(8), e364985169. http://doi.org/10.33448/rsd-v9i8.5169

Fabbro, M. R. C., Salim, N. R., Bussadori, J. C. C., Okido, A. C. C. \& Dupas, G. (2018). Estratégias ativas de ensino e aprendizagem: percepções de estudantes de Enfermagem. Revista Mineira de Enfermagem, 22, e-1138. http://www.doi.org/10.5935/1415-2762.20180067

Fontes, F. L. L., Santana, R. S., Santo, I. M. E., Barros, R. N. S., Maroja, M. C. F. S., Nahum, B. A. P., Granjeiro, K. N. M. M., Sousa, M. S. R., Soares, J. C., Oliveira, I. I., Figueiredo, J. O., Silva, F. J. A., Silva, L. J. G., Costa, A. C. R. R. \& Rodrigues, M. S. (2019). A Enfermagem no ensino superior: estratégias utilizadas pelo enfermeiro docente para melhoria de suas práticas pedagógicas. Revista Eletrônica Acervo Saúde, S18, e435. https://doi.org/10.25248/reas.e435.2019

Fontes, F. L. L., Santana, R. S., Silva, H. L. L., Bezerra, A. M. F. A., Brito, R. G., Oliveira, I. I., Silva, D. S. N., Morais, M. J. A., Ribeiro, R. N., Santo, I. M. B. E., Senna, S. B. B., Melo, C. E. M. \& Milanez, A. L. O. (2020). Atuação do enfermeiro docente no ensino superior: entre o real e o ideal. International Journal of Development Research, 10(5), 35924-35928. https://doi.org/10.37118/ijdr.18795.05.2020

Fontes, F. L. L., Santana, R. S., Soares, J. C., Pereira, R. I. N., Santo I. M. B. E., Sousa, M. S. R., Martins, G. S., Araújo, C. A., Silva, M. J. M., Silva Neta, A. S., Coimbra, G. T., Lopes, M. C. F., Pinho, L. F., Silva, N. K. B \& Costa, A. M. A. S. (2019). Desafios e dificuldades enfrentadas pelo enfermeiro docente para o exercício da docência no ensino superior. Revista Eletrônica Acervo Saúde, S24, e300. https://doi.org/10.25248/reas.e300.2019

Felix, A. M. S. \& Soares, R. A. Q. (2019). Metodologias ativas no ensino de enfermagem em doenças transmissíveis. Revista de Enfermagem da UFPE, 13, e241816. https://doi.org/10.5205/1981-8963.2019.241816

Freitas, C. A. O. \& Santos, A. C. M. (2019). Uso de metodologias ativas no ensino de práticas de Enfermagem. Revista de Enfermagem da UFPE, 13, e241524. https://doi.org/10.5205/1981-8963.2019.241524

Macedo, K. D. S., Acosta, B. S., da Silva, E. B., de Souza, N. S., Beck, C. L. C. \& da Silva, K. K. D. (2018). Metodologias ativas de aprendizagem: caminhos possíveis para inovação no ensino em saúde. Revista Escola Anna Nery, 22(3), e20170435. https://doi.org/10.1590/2177-9465-EAN-2017-0435

Mendonça, A. R., Queluci, G. C., Dias, S. F. C. \& de Souza, V. R. (2017). Estratégias de aprendizagem ativa em Enfermagem. Revista Pró-univerSUS, 8(2), 117-120.

Melo, T. O., Tacahashi, D. S., Freitas, P. P. S. \& Oliveira, P. N. (2014). O olhar do docente acerca dos alunos que trabalham inseridos nas metodologias ativas de aprendizagem. Revista da Faculdade de Ciências Médicas de Sorocaba, 16(3), 134-138.

Mesquita, S. K. C, Meneses, R. M. V. \& Ramos, D. K. R. (2016). Metodologias ativas de ensino/aprendizagem: dificuldades de docentes de um curso de Enfermagem. Revista Trabalho, Educação e Saúde, 14(2), 473-486. http://doi.org/10.1590/1981-7746-sip00114

Mitre, S. M., Batista, R. S., Mendonça, J. M. G., Pinto, N. M. M., Meirelles, C. A. B., Porto, C. P. (2008) Metodologias ativas de ensino-aprendizagem na formação profissional em saúde: debates atuais. Ciência \& Saúde Coletiva, 13(S12), 2133-2144. https://doi.org/10.1590/S1413-81232008000900018

Pascon, D. M., Otrenti, E. \& Mira, V. L. (2018). Percepção e desempenho de graduandos de Enfermagem em avaliação de metodologias ativas. Revista Acta Paulista de Enfermagem, 31(1), 61-70. https://doi.org/10.1590/1982-0194201800010

Rodrigues, R. M. \& Caldeira, S. (2008). Movimentos na educação superior na educação superior, no ensino em saúde e na Enfermagem. Revista Brasileira de Enfermagem, 61(5), 629-636. https://doi.org/10.1590/S0034-71672008000500016

Santos, E. F. (2005). A Enfermagem no espelho: suas concepções sobre a profissão, a formação, a prática profissional e as especialidades. Tese de Doutorado. Instituto de Medicina Social, Universidade do Estado do Rio de Janeiro, Rio de Janeiro.

Santos, T. A. D., Fontes, F. L. L., Bezerra, A. M. F. A., Senna, S. B. B., Luz, A. S., Pereira, R. I. N., Silva, M. A. S., Oliveira, I. I., Santos, J. P. F., Brito, R. G., Sousa, M. I. B., Santos, M. C. S. P., Cruz, D. L., Silva, T. C. \& Silva, H. K. F. (2020). Importância da formação continuada para o exercício da docência do ensino superior na Enfermagem. In: Silva, M. C. A. Impressões sobre o Cuidar de Enfermagem Sistematizado 3. Ponta Grossa: Atena Editora. https://doi.org/10.22533/at.ed.88120230412

Xavier, L. N., Oliveira, G. L., Gomes, A. A., Machado, M. F. A. S. \& Eloia, S. M. C. (2014). Analisando as metodologias ativas na formação dos profissionais de saúde: uma revisão integrativa. SANARE, 13(1), 76-83. 